Pacific Journal of Mathematics

MAXIMAL MODELS IN THE LANGUAGE WITH QUANTIFIER 


\section{MAXIMAL MODELS IN THE LANGUAGE WITH QUANTIFIER “THERE EXIST UNCOUNTABLY MANY",}

\section{J. I. Malitz AND W. N. ReinhardT}

Fuhrken has shown that the language $L_{\omega_{1}}^{Q}$, obtained from first order logic by adding a new quantifier $Q$ and interpreting $Q x$ as "there are at least $\omega_{1} x$ 's such that ..."' is countably compact but not fully compact. The countable compactness is not enough to yield strong analogs of the upward Löwenheim-Skolem theorem, and the amalgamation property. In fact, it is shown that for "most" cardinals $\kappa$, there are structures of power $\kappa$ with countable type that are maximal in the sense of having no proper extensions with the same $L_{\omega_{1}}^{Q}$ theory. From this the failure of the amalgamation property is obtained. There is still the possibility that the model theory of $L_{\kappa}^{Q}$ (with $Q x$ interpreted as "there are at least $\kappa x$ 's such that...') for $\kappa>\omega_{1}$, is more analogous to the model theory of first order logic.

Our investigations of $L_{\omega 1}^{Q}$ turn out to be closely related to questions in first order logic studied by Rabin [13], Keisler [7], and Chang [2], and in the logic of $L_{\omega}^{Q}$ studied by Craig [3], and Keisler [6]. Our development provides some unification of these results and some strengthening (cf. Theorems 2.12 and Corollary 3.5) of Keisler's results on $L_{\omega}^{Q}$ characterizability. Fuhrken's work on the compactness of $L_{\omega_{1}}^{Q}$ appears in [4].

o. Preliminaries and notation. Ordinals are denoted by the letters $\alpha, \beta, \gamma$, and cardinals by $\kappa, \lambda, \mu$ with $m, n$ reserved for finite cardinals. The first uncountable measurable cardinal is denoted by $1^{\text {st }} M C$. We identify each ordinal with the set of smaller ordinals and the cardinals with the initial ordinals. $c X$ will denote the cardinality of $X$. By ${ }^{r} X$ we mean the set of all functions on $Y$ to $X$. $c\left({ }^{2} \kappa\right)$ is written as $\kappa^{2} . \quad{ }^{2} \kappa=\mathrm{U}_{\alpha \in \lambda}{ }^{\alpha} \kappa ;{ }^{2} \kappa=c\left({ }^{2} \kappa\right) . \quad R_{\kappa}$ is the sets of rank less than $\kappa$. $S x=\{t: t \subseteq x\}$.

Sans serif will be used for the nonlogical symbols, $\theta, \varphi, \psi, \sigma$ will denote formulas. We use $u, v$ for variables and $\bar{u}, \bar{v}$ for finite sequences of variables. The type $\tau \Sigma$ of a set $\Sigma$ of formulas is simply the set of nonlogical symbols occurring in $\Sigma$.

Capital German letters will be reserved for structures, and the corresponding Roman letters used for their universes. We also write $|\mathfrak{A}|$ for the universe of $\mathfrak{A}$. All structures are infinite unless explicitly 
assumed finite. The denotation of the symbol $J$ in $\mathfrak{U}$ will be written $J^{\mathfrak{x}}$. The type of $\mathfrak{N}$, written $\tau \mathfrak{R}$, is the set of all nonlogical symbols having denotations in $\mathfrak{A}$. " $\kappa$ admits a structure such that... " means "there is structure $\mathfrak{A}$ such that $c|\mathfrak{U}|=\kappa$ and ...".

An assignment in $\mathfrak{X}$ is a function on the variables taking values in $|\mathfrak{A}|$. If $\varphi$ is a formula, and $z$ is an assignment in $\mathfrak{U}$, we write $\mathfrak{U} \vDash \varphi[z]$ to mean that $\varphi$ is satisfied in $\mathfrak{U}$ under the assignment $z$. We write $\mathfrak{A} \subseteq \mathfrak{B}$ to mean that $\mathfrak{A}$ is a substructure of $\mathfrak{B}$ ( $\mathfrak{B}$ is an extension of $\mathfrak{U}) ; \mathfrak{U}<_{\Gamma} \mathfrak{B}$ means that $\mathfrak{X} \subseteq \mathfrak{B}$ and for for every formula $\varphi \in \Gamma$ and every assignment $z$ in $\mathfrak{X}, \mathfrak{A} \vDash \varphi[z]$ iff $\mathfrak{B} \vDash \varphi[z] ; \quad \mathfrak{U} \equiv_{\Gamma} \mathfrak{B}$ means that for each sentence $\sigma \in \Gamma$, $\mathfrak{U} \vDash \sigma$ iff $\mathfrak{B} \vDash \sigma$. We write $\prec$ and $\equiv$ respectively for $\prec_{\Gamma}$ and $\equiv_{\Gamma}$ in case $\Gamma$ is first order logic, and $\prec_{k}$, $\equiv_{\kappa}$ in case $\Gamma$ is $L_{\kappa}^{Q}$. $T h_{L} \mathfrak{A}=\{\sigma \in L: \mathfrak{X} \vDash \sigma\}$.

The language $L_{\kappa}^{Q}$, defined for $k \geqq \omega$, is obtained from first order logic by the addition of a new quantifier symbol $Q_{k}$ (we will write simply $Q$ if the cardinal is clear or immaterial). In $L_{i}^{Q}, Q u$ has the interpretation "there are at least $\kappa$ u's such that...". Thus in $L_{\kappa}^{Q}$, the assignment $z$ in $\mathfrak{X}$ makes the formula $Q u \varphi$ true in $\mathfrak{X}$ iff $c\{b \in|\mathfrak{U}|$ : $\mathfrak{U} \vDash \varphi[z(u / b)]\} \geqq \kappa$. Let $t \leqq \tau \mathfrak{X}$ and let $\phi \neq V \leqq|\mathfrak{U}|$. Then $\mathfrak{X} \mid(V, t)$ is the $t$-reduct of the substructure of $\mathfrak{A}$ determined by $V$, i.e., if $\mathfrak{B}$ is the substructure of $\mathfrak{U}$ determined by $V, \mathfrak{U} \mid(V, t)$ is the structure (5) with universe $|\mathfrak{B}|$ and type $t$ determined by $R^{\mathbb{E}}=R^{\mathfrak{B}}$ for $R$ in $t$. We write $\mathfrak{U} \mid t$ for $\mathfrak{U} \mid(|\mathfrak{A}|, t)$. If $v$ is a unary relation symbol, then we will write $\mathfrak{A} \mid(v, t)$ for (the relativized reduct) $\mathfrak{A} \mid\left(V^{\mathfrak{A}}, t\right)$. If $\left\langle R_{i}\right.$ : $i \in I\rangle$ is a family of relations on $|\mathfrak{A}|$, then $\left(\mathfrak{U}, R_{i}\right)_{i \in I}$ denotes a structure which results from $\mathfrak{U}$ by extending the type of $\mathfrak{A}$ to include new relation symbols $R_{i}, i \in I$, with $R_{i}^{q}=R_{i}$.

1. In this section we introduce the notion of strongly maximal structure, which encompasses the two maximality notions which most concern us in this paper, those for $L_{\omega}^{Q}$ and $L_{\omega_{1}}^{Q}$. This will be shown in Theorem 1.2 and its partial converse, Theorem 1.10. Also in $\S 1$ are examples of strongly maximal models which are basic to our development (Lemmas 1.3, 1.7 and following remarks).

Definition 1.1. (a) $\mathfrak{U}$ is $L$-maximal iff whenever $\mathfrak{U} \equiv_{L} \mathfrak{B}$ and $\mathfrak{U} \subseteq \mathfrak{B}$ then $\mathfrak{A}=\mathfrak{B}$.

(b) A structure $\mathfrak{X}=\left\langle A, U^{\mathfrak{x}},\left\langle^{\mathfrak{x}}, \cdots\right\rangle\right.$ with $U^{\mathfrak{x}}$ unary and $<^{\mathfrak{x}}$ binary is called strongly maximal iff

(i) any structure $L_{\omega}^{Q}$-equivalent to $\left\langle U^{q},\left\langle^{q}\right\rangle\right.$ is countable and

(ii) whenever $\mathfrak{A} \subseteq \mathfrak{B}, \mathfrak{A} \equiv \mathfrak{B}$, and $U^{\mathfrak{B}}$ is countable then $\mathfrak{A}=\mathfrak{B}$.

For example condition (bi) is satisfied if $\left\langle U^{\mathfrak{x}},\left\langle^{\mathfrak{x}}\right\rangle \cong\langle\omega,\langle\rangle\right.$, as this is $L_{w}^{Q}$-characterizable (up to isomorphism) as an infinite linear 
order in which each element has finitely many predecessors.

THEOREM 1.2. If $\mathfrak{X}$ is strongly maximal, then $\mathfrak{X}$ is both $\omega$-maximal and $\omega_{1}$-maximal.

Proof. That strongly maximal implies $\omega_{1}$-maximal is obvious since in $L_{\omega_{1}}^{Q}$ we can say directly that $U$ is countable. To see that strongly maximal implies $\omega$-maximal it is enough to see that if $\mathfrak{U}$ is a strongly maximal structure with $\mathfrak{A} \subseteq \mathfrak{B}$ and $\mathfrak{A} \equiv{ }_{\omega} \mathfrak{B}$ then $U^{\mathfrak{B}}$ is countable. In Lemmas 1.3 and 1.7 we give examples of strongly maximal models which are basic to our development.

LEMMA 1.3 Let $\omega_{1} \leqq \lambda$. There is a strongly maximal model $\mathfrak{A}$ with $c|\mathfrak{U}|=\omega$ and $c \tau \mathfrak{A}=\lambda$.

REMARK. The case $\lambda \geqq 2^{\omega}$ is immediate from Rabin's result that the complete structure on $\omega$ has no proper elementarily equivalent extension of cardinality $\omega$ (Rabin [13]; see also Malcev [11], Keisler [6], and Chang [2]). The case $\lambda<2^{\omega}$ is implicit in Rabin's proof. The examples we give are different from Rabin's. Chang's argument makes essential use of the complete structure (see remark after Theorem 2.10).

Proof. It is enough to find a countable structure with no countable proper equivalent extensions, as we may realize the structure on $\omega$ and add the relation < required in the definition of strongly maximal.

G. Carpenter, while an undergraduate at Boulder, pointed out the following particularly simple proof. Let $\mathfrak{A}=\langle Q, Z,+, \cdot, D\rangle_{D \in X}$ where $Q$ is the rationals and $X$ is any uncountable set of reals (left Dedekind cuts). In any proper equivalent extension $\mathfrak{B}$ of $\mathfrak{A}$, there must be an infinitesimal $\varepsilon$. In fact since $\mathfrak{B}$ satisfies $\forall x \exists m, n(Z m \wedge Z n \wedge m \cdot x \approx$ $n)$, there must be a nonstandard integer $n^{*} \in Z^{8}$ whose reciprocal is an infinitesimal. But for each $D \in X$, there is $b \in B$ within $\varepsilon$ of $D$, i.e., $(D(b) \wedge \sim D(b+\varepsilon)) \vee(D(b-\varepsilon) \wedge \sim D(b))$. Different $D$ 's get different $b$ 's, so $B$ is uncountable.

LEMMA 1.4. If $\mathfrak{X}$ is a strongly maximal structure with $c|\mathfrak{A}|=\kappa$ and $c \tau \mathfrak{N} \leqq \kappa$ then there is a strongly maximal $\mathfrak{B}$ with finite type and $c|\mathfrak{B}|=\kappa$.

Proof. We may suppose without loss of generality that $\tau \mathfrak{A}$ has $\kappa n$-place relation symbols $R_{n, \alpha}$, so that $\mathfrak{U}=\left\langle\kappa, R_{n, \alpha}^{\mathfrak{q}}\right\rangle_{n \in \omega, \alpha \in \kappa}$. We introduce a new type $t=\left\{S_{n}: n \in \omega\right\}$ where $S_{n}$ is a new relation symbol 
of rank $n+1$. Define $\mathbb{S}$ of type $t \cup\{U,<\}$ by putting $|\mathfrak{S}|=\kappa, U^{\mathbb{E}}$ $u^{\mathscr{Q}},<^{\mathbb{E}}=<^{\mathscr{q}}$, and

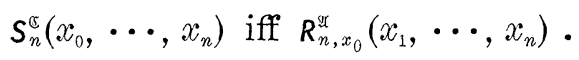

Let $\mathfrak{B}$ be an expansion of $\mathfrak{C}$ with Skolem functions, so that $\mathfrak{B} \subseteq \mathfrak{B}^{\prime}$ implies $\mathfrak{B} \prec \mathfrak{B}^{\prime}$. Clearly $\mathfrak{B}$ has countable type. We claim that $\mathfrak{B}$ is strongly maximal. Suppose $\mathfrak{B} \subseteq \mathfrak{B}^{\prime}, \mathfrak{B} \equiv \mathfrak{B}^{\prime}$, and $c\left(U^{\mathfrak{B}}\right)=\omega$. Define

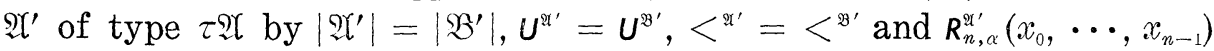
iff $\boldsymbol{S}_{n}^{\mathfrak{B} \prime}\left(\alpha, x_{0}, \cdots, x_{n-1}\right)$. Since $\mathfrak{B} \prec \mathfrak{B}^{\prime}, \mathfrak{Y} \equiv \mathfrak{U}^{\prime}$; since $\mathfrak{B} \subseteq \mathfrak{B}^{\prime}, \mathfrak{U} \subseteq \mathfrak{U}^{\prime}$;

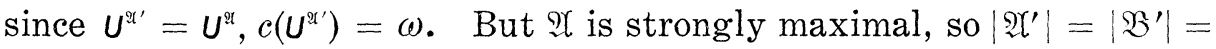
$|\mathfrak{S}|=\kappa$ and $\mathfrak{B}=\mathfrak{B}$.

The reduction to a finite type may be accomplished, in the usual way, by coding everything with a binary relation. We omit the proof.

For the proof of Lemma 1.6 we need the following well known fact.

Lemma 1.5. For each cardinal $\kappa$, if $\lambda=\kappa^{+}$or $\lambda=2^{\kappa}$, then there is a structure $\mathfrak{B}$ whose type is finite and includes a unary predicate $\kappa$, and such that $|\mathfrak{B}|=\lambda, K^{\mathfrak{B}}=\kappa$, and whenever $\mathfrak{B} \subseteq \mathfrak{S}, \mathfrak{B} \equiv \mathfrak{C}$, and $\kappa^{\sqrt{5}}=\kappa$, then $\mathfrak{B}=\mathfrak{E}$.

Proof. For $\lambda=2^{\kappa}$, choose $\mathfrak{B} \cong\langle S \kappa, \kappa, \in\rangle$. For $\lambda=\kappa^{+}$, choose a binary function $F$ so that for $\alpha \in \lambda \sim \kappa, F(\alpha, \cdot)$ enumerates the predecessors of $\alpha$ on $\kappa$. Then take $\mathfrak{B}=\left\langle\kappa^{+}, \kappa, \in, F\right\rangle$.

Lemma 1.6. Let $\lambda=\kappa^{\dagger}$ or $\lambda=2^{\kappa}$. If there is a strongly maximal model $\mathfrak{A}$ with $c|\mathfrak{A}|=\kappa$, then there is a strongly maximal $\mathfrak{B}$ with $c|\mathfrak{B}|=\lambda$ and $c \tau \mathfrak{B} \leqq c \tau \mathfrak{N}+2$.

Proof. Let $\mathfrak{U}$ be a strongly maximal model with $|\mathfrak{U}|=\kappa$. By

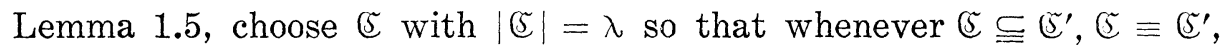

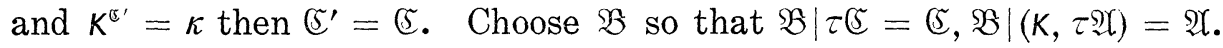
We claim that $\mathfrak{B}$ is the desired strongly maximal structure. For suppose that $\mathfrak{B} \subseteq \mathfrak{B}^{\prime}, \mathfrak{B} \equiv \mathfrak{B}^{\prime}$, and $c\left(\cup^{\mathfrak{g}}\right)=\omega$. Then $\mathfrak{U}^{\prime}=\mathfrak{V}^{\prime} \mid(\kappa, \tau \mathfrak{X}) \equiv$ $\mathfrak{B} \mid(K, \tau \mathfrak{X})=\mathfrak{X}$, so $\mathfrak{X} \subseteq \mathfrak{U}^{\prime}, \mathfrak{X} \equiv \mathfrak{U}^{\prime}$, and $c\left(\mathcal{U}^{\mathfrak{N}}\right)=\omega$. But $\mathfrak{U}$ is strongly maximal, so $K^{\mathfrak{g} \prime}=\kappa$. By choice of $\mathfrak{E}$, we now have $\mathfrak{E}=\mathfrak{C}^{\prime}$, hence $\mathfrak{B}=\mathfrak{B}^{\prime}$.

LEMma 1.7. Let $\lambda=\omega_{1}$ or $\lambda=2^{\omega}$. Then there is a strongly maximal model of cardinality $\lambda$ and finite similarity type.

Proof. Immediate from Lemmas 1.3, 1.6, and 1.4.

REMARK. (a) In the case $\lambda=2^{\omega}$, there is a strongly maximal 
structure which is particularly easy to describe. Namely, $\langle R, Q$, $Z,+, \cdot\rangle$ is such a structure $(R=$ reals $)$, as the proof of Lemma 1.3 shows.

(b) We now give an example of a strongly maximal structure which admits many automorphisms. This will be useful later. Let $T=\bigcup_{n \in \omega}{ }^{n} 2$, so that $\langle T, \subseteq\rangle$ is the full binary tree. Let $L_{n}={ }^{n} 2$, the set of points at the $n$th level. We now define a four place relation $F$ by $F a b x y$ iff $x \leqq a \in{ }^{\omega} 2, y \leqq b \in{ }^{\omega} 2$ and for some $n \in \omega, x, y \in{ }^{n} 2$. Now the structure $\mathscr{T}=\left\langle T \cup{ }^{\omega} 2, T \text {, } \subseteq, L_{n}, F\right\rangle_{n \in \omega}$ is strongly maximal. To see this, suppose $\mathfrak{B} \equiv \mathscr{T}, \mathfrak{B} \supseteqq \mathscr{T}, T^{\mathfrak{B}}$ is countable and $x$ is a new point in $B$. Evidently we may suppose that $x \in T^{\mathfrak{g}}$. Clearly $x \notin L_{n}^{\mathfrak{B}}$ for any $n \in \omega$. Hence $\cup\left\{t \in|\mathscr{T}|: t \subseteq^{\mathscr{B}} x\right\}$ is a maximal branch $b \in{ }^{\omega} 2$. Clearly $\left\{y\right.$ : for some $\left.a \in{ }^{\omega} 2, F^{3} b a x y\right\}$ is uncountable. Indeed, if $F^{\mathfrak{B}} b a x y$ and $F^{\mathfrak{B}} b a^{\prime} x y$ then $\cup\left\{s \in{ }^{\omega} 2: s \subseteq^{8} y\right\}=a=a^{\prime}$. This contradicts the countability of $T^{\mathscr{P}}$. What makes $\mathscr{T}$ useful in constructing examples is that any automorphism of $\langle T, \subseteq\rangle$ induces a unique automorphism of $\mathscr{T}$.

The next two theorems combine the notion of *-theory (as is found in Morley-Vaught [12, §3]) and Fuhrken's normal form [4].

THEOREM 1.8. Every structure $\mathfrak{N}$ of power at least $\kappa$ has an expansion $\mathfrak{A}^{*}$ with $c \tau \mathfrak{A} \mathfrak{H}^{*}=\omega+c \tau \mathfrak{U}$ and with a unary predicate symbol $U \in \tau \mathfrak{Q} *$, such that whenever $\mathfrak{X}^{*} \subseteq \mathfrak{B}$ and $\mathfrak{I}^{*} \equiv \mathfrak{B}$ and $c \cup^{\mathfrak{B}}=\kappa$ then $\mathfrak{U} * \prec_{\kappa+} \mathfrak{B}$.

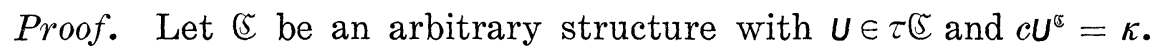
For each formula $\varphi$ of type $\tau \widetilde{C}$ we choose new function symbols $f_{\varphi}$ and $g_{\varphi}$ and corresponding functions on $C$ such that the structure (5' obtained from $(5$ by adjoining these functions is a model of the following sentences:

(i) $\quad \forall \bar{u}\left(\exists \bar{v} \varphi_{\bar{u}}, v \rightarrow \varphi_{\bar{u}}, f_{\varphi}(\bar{u})\right)$

(ii) $\forall \bar{u}\left(\neg Q v v \bar{u}, v \rightarrow \forall v\left(\varphi \bar{u}, v \rightarrow U g_{\varphi}(\bar{u}, v)\right)\right)$

(iii) $\forall \bar{u}, v, v^{\prime}\left(g_{\varphi}(\bar{u}, v) \approx g_{\varphi}\left(\bar{J}, v^{\prime}\right) \rightarrow v \approx v^{\prime}\right)$

where $\bar{u}$ is some enumeration of the free variables of $\phi$ other than $v$.

Now expand the given structure $\mathfrak{N}$ to $\mathfrak{U}_{0}=\left(\mathfrak{U}, U^{\mathfrak{x}_{0}}\right)$ where $U^{\mathfrak{x}_{0}}$ is any subset of $A$ of power $\kappa$. By induction define

$$
\mathfrak{A}_{n+1}=\left(\mathfrak{U}_{n}\right)^{\prime} \quad \text { (where' } \text { is as above) . }
$$

Now $\mathfrak{U}^{*}$ is defined by $\tau \mathfrak{A}^{*}=\cup_{n \in \omega} \tau \mathfrak{A}_{n}$, and $\mathfrak{X}^{*} \mid \tau \mathfrak{A}_{n}=\mathfrak{A}_{n}$. We claim that $\mathfrak{U}^{*}$ has the desired properties. Suppose $\mathfrak{X}^{*} \subseteq \mathfrak{B}, \mathfrak{X}^{*} \equiv \mathfrak{B}$ and $c\left(U^{\sharp)}=\kappa\right.$. We prove by induction on formulas $\phi$ of $L_{\kappa}^{Q+}$ that

$$
\mathfrak{Y}^{*} \vDash \varphi[z] \text { iff } \mathfrak{B} \vDash \varphi[z]
$$


for any assignment $z$ in $A$. For the induction we introduce the following ranking of formulas:

$$
r \varphi= \begin{cases}0 & \text { if } \varphi \text { is atomic } \\ r \theta+1 & \text { if } \varphi \text { is } \neg \theta \\ r \theta_{1}+r \theta_{2}+1 & \text { if } \varphi=\theta_{1} \rightarrow \theta_{2} \\ r \theta+1 & \text { if } \varphi=\exists v \theta \\ r \theta+4 & \text { if } \varphi=Q v \theta .\end{cases}
$$

The induction is straightforward. We consider only the case $\phi=\neg$ $Q_{v} \theta \bar{u} v$. Assume that for every assignment $z$ in $A, \mathfrak{I}^{*} \vDash \theta[z]$ if and only if $\mathfrak{B} \vDash \theta[z]$. Clearly, $\mathfrak{U}^{*} \vDash Q_{v} \theta[z]$ implies $\mathfrak{B} \vDash Q_{v} \theta[z]$. Suppose $\mathfrak{I}^{*} \vDash \neg Q_{v} \varphi[z]$. Since (ii) holds in $\mathfrak{A}^{*}$, we have $\mathfrak{I}^{*} \vDash \forall v\left(\theta(v) \rightarrow U_{g_{\theta}}(\bar{u}\right.$, v)) $[z]$. Hence by induction $\mathfrak{B} \vDash \forall v\left(\theta(v) \rightarrow U g_{\theta}(\overline{\boldsymbol{u}}, v)\right)[z]$. Since $\mathfrak{B} \equiv \mathfrak{U}^{*}$ we have by (iii) that $g_{\theta}^{\mathscr{B}}$ is $1-1$ as a function of $v$. Also, by hypotheses $c\left(U^{\mathfrak{g}}\right)=\kappa$. Hence $\{b \in B: \mathfrak{B} \vDash \theta[z(\mathrm{v} / b)]\}$ is of cardinality $\kappa$. Thus $\mathfrak{B} \vDash \neg Q_{v} \theta[z]$.

THEOREM 1.9. Every structure $\mathfrak{A}$ has an expansion $\mathfrak{A}^{*}$ with ct?* $\mathfrak{A}^{*}=$ $\omega+c \tau \mathfrak{A}$ and a unary predicate symbol $U$ and a binary predicate symbol $<$ in $\tau \mathfrak{2} *$ such that $\left(\cup^{\mathfrak{2 *}},<^{\mathfrak{x}^{*}}\right)$ is $\kappa$-like and whenever $\mathfrak{U}^{*} \subseteq \mathfrak{B}$ and $\mathfrak{X}^{*} \equiv \mathfrak{B}$ and $\left\langle U^{\mathfrak{B}},\left\langle^{\mathfrak{B}}\right\rangle\right.$ is $\kappa$-like then $\mathfrak{X}^{*} \prec_{\kappa} \mathfrak{B}$. (An ordering is $\kappa$-like iff it has cardinality $\kappa$ and each proper initial segment has cardinality less than $\kappa$.)

Proof. The construction of $\mathfrak{2}^{*}$ is as in the preceding theorem except that we choose $\mathfrak{A}_{0}$ so that $\tau \mathfrak{U}_{0}$ includes the binary predicate $<$, and $\left\langle U^{\mathfrak{x}_{0}},\left\langle\mathfrak{x}_{0}\right\rangle \cong\langle\kappa, \in\rangle\right.$. The proof differs only in the treatment of the quantifier $Q$. Since $\mathfrak{U}^{*} \vDash \neg Q_{v} \theta[z]$ means that $\left\{b \in A: \mathfrak{X}^{*} \vDash\right.$ $\theta[z(v / b)]\}$ has cardinality $<\kappa$, using (ii) we see that $\mathfrak{Q}^{*} \vDash \exists u \forall v\left(\theta_{v} \rightarrow\right.$ $\left.\mathbf{g}_{\theta}(\overline{\mathbf{w}}, v)<\mathbf{v}\right)[z]$. Hence by induction $\left.\mathfrak{B} \vDash \exists u \forall v \theta v \rightarrow \mathbf{g}_{\theta}(\overline{\mathbf{w}}, v)<\boldsymbol{u}\right)[z]$. Also (iii) holds in $\mathfrak{U}^{*}$ as well as a sentence saying that the field of $<$ is U. Since $\mathfrak{A}^{*} \equiv \mathfrak{B}$, these sentences are true in $\mathfrak{B}$. Since $\left\langle U^{\mathfrak{P}},\left\langle^{\mathfrak{B}}\right\rangle\right.$ is $\kappa$-like, it follows that $c\{b \in B: \mathfrak{B} \vDash \theta[z(v / b)]\}<\kappa$.

THEOREM 1.10. If $\mathfrak{A}$ is either $\omega_{1}$-maximal, or $\mathfrak{A}$ is $\omega$-maximal

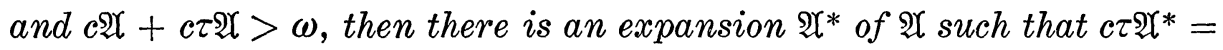
$c \tau \mathfrak{A}+\omega$ and $\mathfrak{U}^{*}$ is strongly maximal.

REMARK. In view of this theorem and Theorem 1.2, we will use maximal to mean strongly maximal.

Proof. First assume that $\mathfrak{A}$ is $\omega_{1}$-maximal of cardinality $\kappa$ and $c \tau \mathfrak{A}=\lambda$. We may assume that $|\mathfrak{A}|=\kappa$. Since any expansion of an 
$\omega_{1}$-maximal structure is $\omega_{1}$-maximal, we may assume that $U,<$ belong to $\tau \mathfrak{A}$ and $U^{\mathfrak{x}}=\omega,<^{\mathfrak{x}}=\epsilon$. We claim that the expansion $\mathfrak{U}^{*}$ of $\mathfrak{A}$ (chosen as in Theorem 1.8 with this $U$ ) is strongly maximal. Clearly $\mathfrak{U}^{*}$ is $\omega_{1}$-maximal, and $c \tau \mathfrak{A}^{*}=\lambda$. Let $\mathfrak{U}^{*} \equiv \mathfrak{B}$ and $\mathfrak{U}^{*} \leqq \mathfrak{B}$ and suppose that $c\left(U^{\mathfrak{B}}\right)=\omega$. We must see that $\mathfrak{U}^{*}=\mathfrak{B}$. But this is clear since $\mathfrak{X}^{*}$ is $\omega_{1}$-maximal and by Theorem $1.8 \mathfrak{U}^{*} \prec_{\omega_{1}} \mathfrak{B}$.

Now suppose that $\mathfrak{A}$ is $\omega$-maximal, of cardinality $\kappa$ and $c \tau \mathfrak{A}=\lambda$ (where $\kappa>\omega$ or $\lambda>\omega$ ). We may assume $|\mathfrak{A}|=\kappa$. Since any expansion of an $\omega$-maximal structure is $\omega$-maximal, we may assume that $U,<$ belong to $\tau \mathfrak{A}$, and $U^{\mathfrak{x}}=\omega$. Moreover, if $\kappa>\omega$ we may assume that the structure given by Lemma $1.7\left(\lambda=\omega_{1}\right)$ is a relativized reduct of $\mathfrak{A}$, and if $\lambda>\omega$ we may assume that the structure given by Lemma $1.3\left(\lambda=\omega_{1}\right)$ is a relativized reduct of $\mathfrak{A}$. In either case $\mathfrak{A}$ has a strongly maximal relativized reduct, say $\mathfrak{E}=\mathfrak{A} \mid(V, \tau \mathfrak{E})$. Now we claim that the expansion $\mathfrak{A}^{*}$ (chosen as in Theorem 1.9 with $U$ as above) is strongly maximal, with type of desired cardinality. Clearly $c \tau^{2 *}=\lambda$, and it is $\omega$-maximal. To see that it is strongly maximal, suppose that $\mathfrak{X}^{*} \leqq \mathfrak{B}, \mathfrak{X}^{*} \equiv \mathfrak{B}$, and $c\left(U^{\mathfrak{g}}\right)=\omega$. We must see that $\mathfrak{2}^{*}=$ $\mathfrak{B}$. Since $\mathfrak{U}^{*}$ is $\omega$-maximal, it is enough to see that $\mathfrak{U}^{*} \prec_{\omega} \mathfrak{B}$. By Theorem 1.9, it suffices to see that $U^{\mathfrak{g}}=U^{\mathfrak{x}}$. Now let $\mathbb{C}^{\prime}=\mathfrak{B} \mid(V, \tau \mathfrak{C})$;

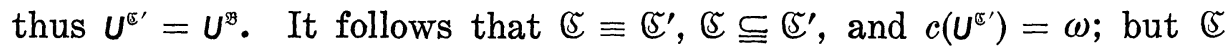
is strongly maximal, so we must have $U^{\Re}=U^{\mathbb{E} \prime}=U^{\mathbb{E}}=U^{\mathfrak{g} *}$ as desired.

2. In this section we investigate the class of cardinals admitting maximal models. The notion of end extension plays an important role.

DEFINITION 2.1. Let $\mathfrak{A}$ be a structure with a binary relation $<^{x}$ on $|\mathfrak{A}|$. We say that an extension $\mathfrak{B}$ of $\mathfrak{A}$ is an end extension of $\mathfrak{U}$ with respect to $<$ iff $x<{ }^{\mathfrak{g}} y \in A$ implies $x \in A$.

LEMMA 2.2. Assume that

(i) $\kappa=\cup M$, where $M$ is a set of infinite cardinals $\nu \in \kappa$ which admit a maximal structure $\mathfrak{B}_{\nu}$ with $\left|\mathfrak{B}_{\nu}\right|=\nu$ and $c \tau \mathfrak{B}_{\nu} \leqq \lambda$ and

(ii) $\langle\kappa, \epsilon\rangle$ has an expansion $\mathfrak{A}$ with $c \tau \mathfrak{A} \leqq \lambda$ and such that $\mathfrak{X}$ has no proper elementary end extensions.

Then there is a maximal structure $\mathfrak{B}$ with $c|\mathfrak{B}|=\kappa$ and $c \tau \mathfrak{B} \leqq \lambda$.

Proof. Let $\mathfrak{A}$ satisfy (ii) and let $\mathfrak{N}^{*}$ be obtained from $\mathfrak{A}$ by adding Skolem functions. We may assume that the $\tau \mathfrak{B}_{\nu}$ are disjoint except for $U$ and $<$, and disjoint from $\tau \mathfrak{A}^{*}$. Choosing new unary predicate symbols $V_{\nu}$, make up $\mathfrak{B}$ so that $\mathfrak{B} \mid \tau \mathfrak{A} \mathfrak{C}^{*}$, and $\mathfrak{B} \mid\left(V_{\nu}, \mathfrak{B}_{\nu}\right)=\mathfrak{B}_{\nu}$. To see that $\mathfrak{B}$ is maximal, suppose that $\mathfrak{B} \subseteq \mathfrak{B}^{\prime}, \mathfrak{B} \equiv \mathfrak{B}^{\prime}$, and $c\left(U^{\mathfrak{B}}\right)=$ 
$\omega$. We must see that $\mathfrak{B}=\mathfrak{B}^{\prime}$. Let $p \in B^{\prime}$. Since $\mathfrak{A}^{*}$ has Skolem functions, $\mathfrak{B} \equiv \mathfrak{B}^{\prime}$, and $\mathfrak{B} \subseteq \mathfrak{B}^{\prime}$, we must have $\mathfrak{A}<\mathfrak{U}^{\prime}=\mathfrak{B}^{\prime} \mid \tau \mathfrak{A}$. Now $\mathfrak{U}$ has no proper elementary end extensions, therefore there is $\nu \in M$ so that $p \in\left|\mathfrak{B}_{\nu}^{\prime}\right|$. Thus $p$ is in the $V_{\nu}$ of $\mathfrak{B}^{\prime}$. Let $\mathfrak{B}_{\nu}^{\prime}=\mathfrak{B}^{\prime} \mid\left(V_{\nu}, \tau \mathfrak{B}_{\nu}\right)$; then $\mathfrak{B}_{\nu} \subseteq \mathfrak{B}_{\nu}^{\prime}, \mathfrak{B}_{\nu} \equiv \mathfrak{B}_{\nu}^{\prime}$, and the cardinality of the denotation of $U$ in $\mathfrak{B}_{\nu}^{\prime}$ is $\omega$. Since $\mathfrak{B}_{\nu}$ is maximal, we must have $\left|\mathfrak{B}_{\nu}^{\prime}\right|=\left|\mathfrak{B}_{\nu}\right|=\nu$, so $p \in$

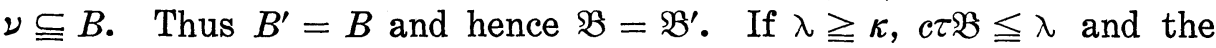
proof is complete. In any case $c \tau \mathfrak{B} \leqq \kappa$, and the proof may be completed by applying Lemma 1.4 .

We now cite a series of results which tell us when the hypothesis (ii) of Lemma 2.2 can be applied. For definitions of measurable and weakly compact, see for example [1].

THEOREM 2.3. (Keisler) $\kappa$ is measurable iff there is a proper elementary end extension of the strusture $\left\langle R_{\kappa}, \in, X\right\rangle_{X \equiv R_{n}}$.

Proof. See Keisler [7, Corollary 3.8]. We indicate a more direct argument for the proof from right to left. Let $\mathfrak{B}$ be the given extension, and $p \in B \sim \kappa$. Then $D=\left\{X \subseteq \kappa: p \in{ }^{\mathfrak{g}} X^{\mathfrak{B}}\right\}$ is a $\kappa$-complete nonprincipal ultrafilter on $\kappa$. As in [7], an ultrapower of $\left\langle R_{\kappa}, \in, X\right\rangle_{X \subseteq R_{\kappa}}$ on a $\kappa$-complete ultrafilter establishes the converse.

In [9], Keisler and Silver prove that if $\kappa$ is strongly inaccessible, then $\kappa$ is weakly compact iff for every $S \subseteq R_{\kappa},\left\langle R_{\kappa}, \in, S\right\rangle$ has a proper elementary end extension. (The result first appeared without proof in Keisler [5]). The next Theorem is an easy strengthening of this.

THEOREM 2.4. $\kappa$ is weakly compact and strongly inaccessible iff for every $S \subseteq R_{\kappa},\left\langle R_{\kappa}, \in, S\right\rangle$ has a proper elementary end extension.

REMARK. In case $\kappa$ is inaccessible, there is a relation $E$ on $\kappa$ such that $\left\langle R_{\kappa}, \epsilon\right\rangle \cong\langle\kappa, E\rangle$ and $E$ is compatible with $\in$ (i.e., $x E y$ implies $x \in y$ ); from this it is easy to see that the two preceding theorems do give conditions assuring that $\langle\kappa, \epsilon\rangle$ has an expansion with no proper elementary end extensions.

Proof of Theorem 2.4. From Keisler-Silver [9], we need only show that $\kappa$ is strongly inaccessible if the right hand side holds. To see that we cannot have $\nu<\kappa \leqq 2^{\nu}$, let $S$ be a $1-1$ function on $\kappa$ into $R_{\nu+1}$. Now in any elementary end extension $\mathfrak{B}, R_{\nu+1}^{\mathfrak{g}}=R_{\nu+1}$ so $\left(R_{\nu+1} \cap \text { range } S\right)^{\mathfrak{g}}=(\text { range } S)^{\mathfrak{B}}=$ range $S$. But if $\mathfrak{B}$ is a proper extension, there must be a point $p \notin \kappa$ which is an ordinal in the sense of $\mathfrak{B}$, contradiction. To see that $\kappa$ must be regular, suppose 
$S: \nu \rightarrow \kappa$ is cofinal and get a contradiction.

Lemma 2.5. If $\lambda=\cup \lambda$ is singular, then there is a structure $\mathfrak{A}$ with countable type and universe $\lambda$ such that $\mathfrak{i}$ has no proper elementary end extensions.

Proof. Let $\kappa=$ cofinality of $\lambda$, and let $F: \kappa \rightarrow \lambda$ be cofinal. Clearly $\langle\lambda, \in, F\rangle$ has no proper elementary end extensions.

Definition 2.6. $\mathfrak{U}=\left\langle R_{\kappa} . \epsilon\right\rangle$ is said to have the $\Pi_{!}^{1}$ refiection property iff whenever $a \in R_{\kappa}$ and $\varphi(a)$ is a $\Pi_{1}^{1}$ sentence (i.e., universal second order) holding in ( $\mathfrak{A}, a)$, there is an $a \in R_{\kappa}$ such that $\varphi(a)$ holds in $\left\langle R_{\alpha}, \in, a\right\rangle$.

Theorem 2.7 (Keisler-Silver). Suppose that $\left\langle R_{\kappa}, \epsilon\right\rangle$ is a model of $Z F$, and cofinality of $\kappa\rangle \omega$. If $\mathfrak{U}=\left\langle R_{\kappa}, \epsilon\right\rangle$ fails to have the $I_{1}^{1}$ reflection property, then $\mathfrak{X}$ has no proper elementary end extension.

REMARK. Keisler and Silver prove Theorem 2.7 for arbitrary well-founded models $\langle A, \epsilon, S\rangle$ of $Z F C$ allowing $S$ in the replacement schema; the definition of ' $\Pi_{1}^{1}$ reflection property' is then slightly more complicated.

\section{Proof. See Keisler-Silver [9].}

DEFINITION 2.8. $\kappa$ is said to have the tree property iff whenever $\left\langle T,\langle\rangle\right.$ is a tree such that $T=\bigcup_{\alpha<x} T_{\alpha}$ (where $x \in T_{\alpha}$ iff $\{y \in T: y<x\}$ has order type $\alpha$ ) and for each $\alpha, 0<c T_{\alpha}<\kappa$, then there is a branch $B \subseteq T\left(\left\langle B,<_{B}\right\rangle\right.$ linearly ordered) which meets each $T_{\alpha}$.

THEOREM 2.9. If $\kappa$ is regular and $\kappa$ does not have the tree property, then $\kappa$ admits a structure with finite type which has no proper elementary end extensions.

Proof. Let $\left\langle T,\left\langle^{T}\right\rangle\right.$ be a tree which provides a counterexample to the tree property for $\kappa$. Thus we have $T=\bigcup_{\alpha<\kappa} T_{\alpha}$ with $0<$ card $T_{\alpha}<\kappa$, where $T_{\alpha}$ is the set of points $x$ in $T$ such that the order type of $\left\{y \in T: y<^{T} x\right\}$ is $\alpha$. By the regularity of $\kappa$, we may assume that $T=\kappa$ and that the partial order $<^{T}$ is so arranged that $x \in T_{\alpha}, y \in T_{\beta}$, and $a \in \beta$ implies $x \in y$. We define an expansion $\mathfrak{X}$ of the given tree with new symbols e, $E$ by putting $e^{T}=\epsilon$ (usual order on $\kappa$ ) and $E^{T}$ the equivalence relation on $\kappa$ defined by $x E y$ iff for some $\alpha \in \kappa, x, y \in$ $T_{\alpha}$. We claim that $\mathfrak{i}$ has no proper elementary end extensions. For 
suppose $\mathfrak{A} \prec \mathfrak{B}$ and $\mathfrak{B}$ is an end extension. Then the following formulas are valid in $\mathfrak{B}$ because they are valid in $\mathfrak{A}$.

(1) $u<v \rightarrow$ uev

(2) uEv $\wedge v<w \rightarrow$ vew

(3) vev $\rightarrow \exists w(w E u \wedge w<v)$.

Now (1) assures that if $y \in T$ and $x<^{\mathfrak{B}} y$, then $x \in T$ also. Similarly (2) assures that if $y \in T$ and $x E^{\mathbb{B}} y$ then $x \in T$ also since for some $z \in T, y \mathrm{e}^{\mathfrak{g}} z$ and hence $x \mathrm{e}^{\mathfrak{y}} z$. Thus (3) says that if $x \in T_{\alpha}$ and $x \mathrm{e}^{\mathfrak{g}} y$, then $T_{\alpha} \cap\left\{z \in T: z<^{\mathbb{B}} y\right\} \neq 0$. Since any new point $p \in B \sim T$ has $x \mathrm{e}^{\mathfrak{B}} p$ for every $x \in T$, we see that the predecessors of $p$ determine a branch through $T$, contrary to the choice of $T$.

THEOREM 2.10. If $\lambda<1^{\text {st }} M C$, then there is a maximal model $\mathfrak{A}$ with $|\mathfrak{A}|=\lambda$ and $c \tau \mathfrak{A} \leqq 2^{\lambda}$.

Proof. We prove this by induction on $\kappa$. The case $\lambda=\omega$ is Lemma 1.3. Suppose that $\lambda$ is a successor cardinal, say $\lambda=\kappa^{+}$, and $\kappa$ has a maximal structure $\mathfrak{A}$ with $c \tau \mathfrak{A} \leqq 2^{\kappa}$. Since $2^{\kappa} \leqq 2^{\lambda}$, we apply Lemma 1.6 and obtain a maximal structure $\mathfrak{B}$ on $\lambda$ with $c \tau \mathfrak{B} \leqq 2^{\kappa} \leqq$ $2^{\lambda}$ as desired.

Now suppose that $\lambda=\cup \lambda$, and $\kappa \in \lambda$ implies that there is a maximal structure on $\kappa$ with type $\leqq 2^{\kappa} \leqq 2^{\lambda}$. By Theorem 2.3 there is a structure $\mathfrak{U}$ on $\lambda$ with no proper elementary end extensions, and $c \tau \mathfrak{A} \leqq 2^{\lambda}$. Thus Lemma 2.2 applies and we conclude that there is a maximal structure $\mathfrak{B}$ on $\lambda$ with $c \tau \mathfrak{A} \leqq 2^{\lambda}$.

REMark. Assuming the $G C H$, Theorem 2.10 for $\lambda<1^{\text {st }}$ uncountable inaccessible follows easily from results in Rabin [13]. Theorem 2.10 may be proved directly (no $G C H$ ) by a slight modification of Chang's argument in [2].

COROLlaRy 2.11. If $\lambda<1^{\text {st }} M C$ then there is a maximal model

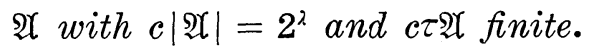

Proof. Use Lemmas 1.6 and 1.4.

The next theorem summarizes our positive results for cardinals admitting maximal models with countable type.

THEOREM 2.12. Let $M$ be the set of cardinals $\kappa$ such that there is a maximal structure on $\kappa$ with finite type. Then $M$ has the following closure properties:

(a) $\omega_{1} \in M$ 
(b) if $\lambda<1^{\text {st }} M C$, then $2^{\lambda} \in M$

(c) if $\lambda \in M$ then $\lambda^{+} \in M$

(d) if $\lambda=U(\lambda \cap M)$ and $\lambda$ singular, then $\lambda \in M$

(e) if $\lambda=\cup(\lambda \cap M)$ and $\lambda$ does not have the tree property, then $\lambda \in M$

(f) if $\lambda$ is strongly inaccessible and not weakly compact, then $\lambda \in M$.

Proof. The first two are restatements of Lemma 1.7 and Corollary 2.11 respectively. The "successor case" lemma (Lemma 1.6) immediately yields (c). Each of (d), (e), and (f) is obtained by use of the lemma for the limit case (Lemma 2.2), together with the lemma for reducing the type (Lemma 1.4); the hypothesis ii) of Lemma 2.2 is obtained (respectively for $\mathrm{d}, \mathrm{e}, \mathrm{f}$ ) by Lemma 2.5, Theorem 2.9, and Theorem 2.4.

REMARK. Let $\theta$ be the first inaccessible, first Mahlo cardinal [cf. 10], etc. (These $\theta$ have the property that $\left\langle R_{\theta}, \epsilon\right\rangle$ fails to have the $\Pi_{1}^{1}$-reflection property where $R_{\theta}$ is the sets of rank $<\theta_{0}$ ) Using the Keisler-Silver Theorem 2.7, our proofs show that $\mathfrak{U}=\left\langle R_{\theta}, \omega, \epsilon\right\rangle$ is weakly maximal in the following sense:

$$
\mathfrak{U} \prec \mathfrak{B} \text { and } c\left(U^{\mathfrak{B}}\right)=\omega \rightarrow \mathfrak{U}=\mathfrak{B} \text {. }
$$

This gives maximal models of power $\theta$ which have simpler descriptions than the models given by the proof of Theorem 2.1.

Definition 2.13. We say that a cardinal $\theta$ has the $\kappa$ end extension property iff every expansion $\mathfrak{A}$ of $\langle\theta,\langle\rangle$ with $c \tau \mathfrak{X}=\kappa$ has a proper end elementary extension (with respect to the usual order $<$ on $\theta$ ).

It is clear from earlier lemmas that a cardinal with the end extension property must be "large" (weakly inaccessible, etc.).

COROLLARY 2.14. Suppose that $\kappa \leqq 1^{\text {st }} M C$, and $\kappa$ admits no maximal model with countable type. Let $\nu=\cap\left\{\mu: 2^{\mu}>\kappa\right\}$ (thus $\nu \leqq$ $\left.\kappa<2^{\nu}\right)$. Then there is a cardinal $\theta, \nu \leqq \theta \leqq \kappa$, such that $\theta$ has the $\kappa$ end extension property (and hence $\theta$ admits no maximal model with type of cardinality $\leqq \kappa)$.

Proof. If $\mu<\nu, \mu$ admits a maximal structure with type of cardinality $\leqq \kappa$. Let $\theta$ be the first cardinal such that $\nu \leqq \theta \leqq \kappa$ and $\theta$ admits no maximal model $\mathfrak{X}$ with $c \tau \mathfrak{H} \leqq \kappa(\kappa$ is such a cardinal by the lemma on reducing types, Lemma 1.4). We claim that $\theta$ has the $\kappa$ 
end extension propərty. Let $\mathfrak{U}$ be any structure on $\theta$ with $c \tau \mathfrak{A} \leqq \kappa$. Choose an expansion $\mathfrak{B}$ of $\mathfrak{P}$ so that $\mathfrak{B} \mid\left(V_{\alpha}, \tau_{\alpha}\right)$ is a maximal model on $\alpha$ (for each cardinal $\alpha<\theta$ ), and $\mathfrak{B}$ includes Skolem functions for $\mathfrak{A}$. Now $\mathfrak{B}$ is not maximal, so $\mathfrak{B}$ has an extension $\mathfrak{C}$. Evidently $\mathfrak{S}\left|\left(V_{\alpha}, \tau_{\alpha}\right)=\mathfrak{B}\right|\left(V_{\alpha}, \tau_{\alpha}\right)$, so $\mathfrak{B} \mid \tau \mathfrak{N}$ must be a proper end elementary extension of $\mathfrak{N}$ as desired.

Theorem 2.15. Suppose that $c|\mathfrak{X}|=\kappa, c \tau \mathfrak{X}=\lambda$, and that one of the following holds:

(1) $\kappa>1^{\text {st }} M C$

(2) $\kappa$ is weakly compact (including the case where $\kappa=\omega$ ) and $\lambda \leqq \kappa$.

Then $\mathfrak{i}$ is not maximal.

Proof. By Theorem 1.2 it suffices to show that $\mathfrak{2}$ is not $\omega_{1}$-maximal. The case $\kappa=\omega$ follows easily from the countable compactness of $L_{i: 1}^{Q}$, (Fuhrken [4]; see also Keisler [8]) using the method of diagrams.

The case where $\kappa$ is weakly compact and $\kappa>\omega$ (and hence $\kappa>\omega_{1}$ ) is proved in the same manner, but using the weak compactness of $L_{\kappa, \kappa}$ and the fact that every sentence of $L_{\omega_{1}}^{Q}$ is logically equivalent to a sentence of $L_{\kappa, \kappa}$.

In case $\kappa>\mu=1^{\text {st }} M C$, the ultrapower $\mathfrak{N}_{D}^{\prime \prime}$ provides a proper $L_{\mu}$ extension of $\mathfrak{i}$ (and hence a proper $L_{\omega_{1}}^{Q}$ extension) whenever $D$ is a $\mu$-complete nonprincipal ultrafilter.

COROLIARY $2.16(G C H)$. Let $M$ be as in Theorem 2.12. Then $\lambda \in M$ iff

(i) $\lambda$ is not weakly compact, and

(ii) $\omega_{1} \leqq \lambda<1^{\text {st }} M C$.

Proof. The "only if" direction is immediate from Theorem 2.12 and the "if" direction from Theorem 2.15.

3. In this section we discuss characterizability; Theorem 3.3 gives a connection between maximal models and structures characterizable up to isomorphism. This is used to obtain results of Keisler (Corollary 3.4) and some improvements (for example Corollary 3.5).

DEFINITION 3.1. Let $L$ be any language which includes first order logic. A structure $\mathfrak{O}$ is $L$-characterizable iff for all $\mathfrak{B}, \mathfrak{U} \equiv_{L} \mathfrak{B}$ implies $\mathfrak{U} \cong \mathfrak{B}$.

Definition 3.2. A structure $\mathfrak{U}$ is called firm iff $\mathfrak{U}$ is isomorphic 
to no proper substructure of itself.

THEOREM 3.3. Let $L$ be any language which includes the first order predicate calculus.

(a) If $\mathfrak{A}$ is L-maximal, $(\mathfrak{A}, \alpha)_{a \in A}$ is L-characterizable and $\mathfrak{A}$ is firm.

(b) If $\mathfrak{Z}$ is L-characterizable and firm, then $\mathfrak{U}$ is L-maximal. (Thus $\mathfrak{i}, a)_{a \in A}$ is $L$-maximal iff it is $L$-characterizable.)

Proof. (a) Let $\mathfrak{X}$ be $L$-maximal. If $\left(\mathfrak{B}, b_{a}\right)_{a \in A} \equiv_{L}(\mathfrak{H}, \alpha)_{a \in A}$, then $\mathfrak{U}^{\prime}=\mathfrak{B} \mid\left\{b_{a} \mid a \in A\right\} \cong \mathfrak{U}$. Thus $\mathfrak{B}$ is an extension of $\mathfrak{Y} \mathfrak{X}^{\prime}$. But $\mathfrak{X}$ (and hence $\left.\mathfrak{X}^{\prime}\right)$ is $L$-maximal, so $\mathfrak{B}=\mathfrak{U}^{\prime}$. It follows that the map $a \rightarrow b_{a}$ is an isomorphism, so ( $\mathfrak{U}, a)_{a \in A}$ is $L$-characterizable. Finally, if $\mathfrak{U} \cong$ $\mathfrak{B} \leqq \mathfrak{A}$, then $\mathfrak{B} \equiv{ }_{L} \mathfrak{A}$, and $\mathfrak{B}$ is $L$-maximal, so $\mathfrak{B}=\mathfrak{A}$. Thus $\mathfrak{A}$ is firm.

(b) Suppose $\mathfrak{A} \subseteq \mathfrak{B}$ and $\mathfrak{U} \equiv_{L} \mathfrak{B}$. If $\mathfrak{U}$ is $L$-characterizable, then $\mathfrak{B} \cong_{f} \mathfrak{A}$. But then if $\mathfrak{U}$ is firm, so is $\mathfrak{B}$, and $f$ is an endomorphism of $\mathfrak{B}$. Therefore $f$ is an automorphism, and hence $\mathfrak{Z}=\mathfrak{B}$. This shows that $\mathfrak{U}$ is $L$-maximal.

CoRollary 3.4 (Keisler). If $\kappa<1^{\text {st }} M C$ then the (complete) structure $\langle\kappa, R\rangle_{R \subseteq 2_{\kappa}}$ is $L_{\omega}^{Q}$-characterizable.

Proof. Immediate from Theorem 2.10 and Theorem 3.3 using the fact that any expansion of a maximal model is maximal. (One could also argue directly as in the proof of Theorem 2.10 without appeal to Theorem 3.3.)

Remarks. 1. From Corollary 3.4 and Theorem 1.2 and 1.10 we can deduce Theorem 2.10. Keisler's proof of Corollary 3.4 [8] uses several complicated theorems on ultraproducts.

2. Keisler's proof [8] is given for the language $L$ with quantifiers $Q_{\mu}$ for $\mu$ measurable, and shows that the complete structure on any cardianl $\kappa$ is $L$-characterizable. It is easy to generalize the examples in Lemmas 1.3 and 1.7 so as to yield maximal models for $L_{i+}^{2+}$. For example, $\left\langle R_{(\ell+1)}, \in, 0\right\rangle$ is $L_{\mu_{+}}^{Q}$-maximal, and, if $S=\{y \subseteq \mu: c y<\mu\}$, $\left\langle\mu \cup S, \mu, \in, 0, X_{\alpha}\right\rangle_{\alpha \in u^{+}}+$with $X_{\alpha}$ distinct has no $L_{k^{2}}$-equivalent extensions $\mathfrak{B}$ with $c\left(U^{\mathfrak{g}}\right)=\mu$. (Proof. The schema

$$
\forall \underset{v}{\exists} \underset{v<w}{\forall}\left(u \in v \longleftrightarrow X_{\alpha}(v)\right)
$$

holds, and in any proper $L_{i l}^{Q}$-equivalent extension, there must be a point $\beta$ greater than all $\gamma \in \mu$. If $x_{\alpha}$ represents $X_{\alpha}$, the $x_{\alpha}$ are distinct.) In this manner one can obtain the rest of Keisler's result by our 
methods.

We also get some improvements of Keisler's results from Theorem 2.14. The improvements consist in reducing $c \tau \mathfrak{B}$ (where $\mathfrak{B}$ is the characterized structure) from $2^{\kappa}$ to $\kappa$. For example,

Corollary $3.5(\mathrm{GCH})$ If $\kappa<1^{\text {st }} M C$ and $\kappa$ not weakly compact, then every structure $\mathfrak{A}$ with $c|\mathfrak{X}|=\kappa$ is the reduct of an $L_{\omega}^{Q}$-character-

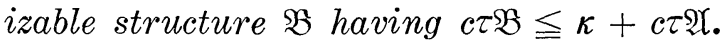

REMARK. The case $\kappa<1^{\text {st }}$ uncountable inaccessible was obtained by Craig and Hanf (also assuming GCH) [3a].

REMARK. One cannot improve the condition on the type of $\mathfrak{B}$ in Corollary 3.5, as is easy to see using the downward Lowenheim-Skolem theorem for $L_{\omega}^{Q}$. On the other hand, it is known that every cardinal admits a firm structure with countable type (səe P. Vopěnka, A. Pultr, Z. Hedrlin, [14]).

4. In this section we use maximal models to show the failure of the amalgamation property for $L_{\omega_{1}}^{Q}$ and the nonexistence of universal models.

Definition 4.1. We say that a triple of structures $\mathfrak{X}, \mathfrak{B}, \mathfrak{Y}^{\prime}$ (all of the same similarity typa) with $\mathfrak{X}^{\prime} \prec_{L} \mathfrak{A}, \mathfrak{B}$ can be $L$-amalgamated in case there is a structure $\mathfrak{C}$, and $L$-embeddings $f: \mathfrak{A} \rightarrow \mathfrak{C}, g: \mathfrak{B} \rightarrow \mathfrak{C}$, such that $f(x)=g(x)$ for $x \in\left|\mathfrak{X}^{\prime}\right|$.

We give two proofs of the failure of amalgamation for $L_{\omega_{1}}^{Q}$. The first (Theorem 4.2) uses the downward Löwenheim-Skolem property of $L_{\omega_{1}}^{Q}$, and the second uses the obvious $L_{\omega_{1}}^{Q}$-analogue of a criterion of Tarski for elementary substructure (Lemma 4.3).

THEOREM 4.2. Suppose that any structure $\mathfrak{U}$ of finite type and cardinality $\geqq \kappa$ has an L-substructure of power $\kappa$. Suppose further, that there is an L-maximal structure of finite type and of cardinality $\lambda$ with $2^{\lambda}>2^{\kappa}$. Then there is a triple $\mathfrak{U}^{\prime} \prec_{L} \mathfrak{A}, \mathfrak{B}$ which cannot be amalgamated.

Proof. Let $M$ be a set of structures each having the same finite type, and such that

(1) $\mathfrak{U} \in M$ implies $c \mathfrak{U}=\lambda$

(2) $\quad c M=\left(2^{\kappa}\right)^{+}$

(3) $\mathfrak{N}, \mathfrak{B} \in M$ and $\mathfrak{V} \neq \mathfrak{B}$ implies $\mathfrak{A} \equiv_{L} \mathfrak{B}$ and $\mathfrak{A} \not \mathfrak{B}$ and $\mathfrak{A}$ is $L$-maximal. 
To obtain such an $M$, let $\mathfrak{U}_{0}$ be $L$-maximal of power $\lambda$ and finite type. Clearly, there are $2^{\lambda}$ nonisomorphic expansions of $\mathfrak{N}_{0}$ that can be obtained by adding one new binary relation to $\mathfrak{A}_{0}$, and any expansion of $\mathfrak{U}_{0}$ is $L$-maximal. Since there are at most $2^{\omega}$ complete extensions of $T h_{L} \mathfrak{A}_{0}$, we can take $M$ to be any set of $\left(2^{\kappa}\right)^{+}$of these expansions which are $L$-equivalent.

For each $\mathfrak{X} \in M$ choose $\mathfrak{X}^{\prime} \prec_{L} \mathfrak{A}$ with card $\mathfrak{U}^{\prime}=\kappa$ (by the downward Löwenheim-Skolem property). Since there are at most $2^{\kappa}$ nonisomorphic $\mathfrak{\mathfrak { X } ^ { \prime }}$, there must be $\mathfrak{X}, \mathfrak{B} \in M$ with $\mathfrak{U} \not \models \mathfrak{B}$ but $\mathfrak{U}^{\prime} \cong \mathfrak{B}^{\prime}$. We may assume that $\mathfrak{X} \prime^{\prime}=\mathfrak{B}^{\prime}$ and that $|\mathfrak{A}| \cap|\mathfrak{B}|=\left|\mathfrak{U}^{\prime}\right|$. Then clearly there is no amalgamation for the triple $\mathfrak{X}^{\prime} \prec_{L} \mathfrak{N}, \mathfrak{B}$.

Lemma 4.3. Suppose that $\mathfrak{Y} \subset \mathfrak{B}$, and that the following two conditions are satisfied for all formulas $\theta$ of $L_{\omega_{1}}^{Q}$ (with free variables $\left.v_{0}, v_{1} \cdots, v_{n}\right)$ and all $a_{1}, \cdots, a_{n} \in|\mathfrak{P}|:$

(i) if there is $b \in|\mathfrak{B}|$ such that $\mathfrak{B} \vDash \theta\left[b, a_{1}, \cdots, a_{n}\right]$, then there is $a_{0} \in|\mathfrak{A}|$ such that $\mathfrak{B} \vDash \theta\left[a_{0}, a_{1}, \cdots, a_{n}\right]$

(ii) if there are uncountably many $b \in|\mathfrak{B}|$ such that $\mathfrak{B} \vDash \theta\left[b, a_{1}\right.$, $\left.\cdots, a_{n}\right]$, then there are uncountably many $c \in|\mathfrak{U}|$ such that $\mathfrak{B} \vDash \theta\left[c, a_{1}\right.$, $\left.\cdots, a_{n}\right]$.

Then $\mathfrak{X} \prec_{\omega_{1}} \mathfrak{B}$.

Proof. By induction on formulas exactly as in the first order case.

LEMMA 4.4. Let $f: \stackrel{\omega}{2} \rightarrow 2$. Then there is a unique automorphism $g$ of $\mathscr{T}$ (the structure defined in Remark $b$ after Lemma 1.7) such that for all $n$ and all $x \in|\mathscr{T}|$.

$$
(g x)_{n}=\left\{\begin{array}{l}
x_{n} \text { if } f(x \backslash n)=0 \\
1-x_{n} \text { if } f(x \backslash n)=1 \text { (i.e., twist where } f=1 \text { ). }
\end{array}\right.
$$

(We write $x \backslash n$ for the restriction of the sequence $x$ to $n$.)

Proof. Clearly, $g$ is $1-1$ and onto; it is also an automorphism since $x \leqq y$ iff $g(x) \subseteq g(y)$, and any automorphism of $\left\langle{ }^{*} 2 \cup^{\omega} 2, \subseteq\right\rangle$ is an automorphism of $\mathscr{T}$.

LEMMA 4.5. Given distinct branches $a, b_{1}, \cdots, b_{m} \in{ }^{\omega} 2$ there is an automorphism $g$ on on $\mathscr{T}$ moving $a$ and keeping $b_{1}, \cdots, b_{m}$ fixed. Moreover there is an $n$ such that for all $x(g x)_{p}=x_{p}$ whenever $p \neq n$.

Proof. Choose $n \in \omega$ such that if $a \backslash m=b_{i} \backslash m$ then $m<n$. Now set $f:{ }^{\omega} 2 \rightarrow 2$ constantly 0 except at $a \backslash n$, and apply Lemma 4.4 to get the desired automorphism. 
THEOREM 4.6. There is a triple $\mathfrak{X}^{\prime} \prec_{\omega_{1}} \mathfrak{X}, \mathfrak{B}$ for which the $L_{\omega-}^{Q}$ amalgamation property fails, and card $\mathfrak{U}^{\prime}=$ card $\mathfrak{U}=2^{\omega}$, and $\mathfrak{U} \cong \mathfrak{B}$.

Proof. Let $\mathfrak{X}=(\mathscr{T}, R)$ where $R \subseteq{ }^{\omega} 2, x \in R$ iff $x_{n}$ is eventually 1. Let $\mathfrak{B}=(\mathscr{T}, S)$, where $S=R \cup\{c\}$, where $c$ is the constant sequence with value 0 . Let $\mathscr{X}$ be the substructure of $\mathfrak{A}$ obtained by omitting $c$.

First we observe that $\mathfrak{A} \cong \mathfrak{B}$. In fact, the automorphism of $\mathscr{T}$ induced (via Lemma 4.4) by the function $f:{ }^{\omega} 2 \rightarrow 2$ given by

$$
f(x)= \begin{cases}1 & \text { if } x \text { is constantly } 1 \\ 0 & \text { otherwise }\end{cases}
$$

is such an isomorphism. If $g$ is the induced automorphism, and $d$ is the constant function with value 1 , then $d \in R$ and $g(d)=c$. If $b \in{ }^{\omega} 2$ and $b \neq d$, then $b, g(b)$ differ at only finitely many places, so $b \in R$ implies $g(b) \in R$. Similarly if $b \notin R, g(b) \notin R$. Thus $g$ : $\mathfrak{U} \cong \mathfrak{B}$.

Next we observe that $\mathfrak{U}^{\prime} \prec_{\omega_{1}}$ 思. We apply Lemma 4.3, for which it is sufficient to see that there is an automorphism of $\mathfrak{N}$ which moves $c$ but keeps fixed any preassigned finite set of elements of $\left|\mathfrak{Z}^{\prime}\right|$. This follows easily from Lemma 4.5. The same argument shows that if $\sqrt{5}$ is the substructure of $\mathfrak{T}$ obtained by omitting $d$, then $\left(\mathfrak{S} \prec_{\omega_{1}} \mathfrak{H}\right.$. Since $g\left(\mathfrak{I} \mathfrak{I}^{\prime}\right)=\mathfrak{S}$, and $g \mathfrak{U}=\mathfrak{B}$, it is evident that $\mathfrak{U}^{\prime} \prec_{\omega_{1}} \mathfrak{B}$.

Since $\mathscr{T}$ is maximal, so are $\mathfrak{Y}, \mathfrak{B}$. Hence if $\mathfrak{D}$ amalgamated $\mathfrak{N}, \mathfrak{B}$ we would have $\mathfrak{U}=\mathfrak{D}=\mathfrak{B}$, which is impossible.

REMARK. In general there are neither universal nor homogeneous models in $L_{\omega_{1}}^{Q}$ (cf. Morely-Vaught [12] for the definition of homogeneous and universal). For example, it is easily seen that the $L_{\omega_{1}}^{Q}$ theory of $\langle A, C\rangle$ where $C$ is a countable unary predicate cannot admit homogeneous models of any power. Also if $T$ is a complete theory with two nonisomorphic maximal models of power $\kappa$, or at least one maximal model of power $<\kappa$, then $T$ admits no universal models of power $\kappa$.

REMARK. In [4a, p. 125], G. Fuhrken has observed that there are no maximal models in $L_{\kappa}^{Q}$ provided that $\kappa$ is not cofinal with $\omega$ and whenever $\mu<\kappa$ then $\mu^{\prime \prime}<\kappa$. To see this apply Theorem 1.9 with $\mathfrak{B}$ the ultraproduct $\left(\mathfrak{U}^{*}\right)_{D}^{\infty}$ and use the fact that $(\kappa,<)_{D}^{\omega}$ is $\kappa$-like. Moreover, the referee pointed out that conversely if $c f \kappa=\omega$ or if for some $\lambda \in \kappa$ we have $\lambda^{\omega} \geqq \kappa$, then there are $L_{\kappa}^{Q}$-maximal structures of power $\kappa$ with countable type. This follows from Theorem 3.3 and Fuhrken $[4 \mathrm{a}, \mathrm{p} .124,(6)]$. 
1. Does the amalgamation property hold in $L_{\omega_{2}}^{Q}$ (or more generally for $\lambda>\omega_{1}$ and ef $\left.\lambda \neq \omega\right)$ ?

2. In Theorem 4.6, could we take $\mathfrak{Y}, \mathfrak{U}, \mathfrak{B}$ all isomorphic or all of power $\omega_{1}$ ?

3. If $L$ is countably compact but not $\omega_{1}$ compact, and $L$ includes first order logic, must the quantifier "there exist at least $\omega_{1} x$ 's" be definable in $L$ ?

It has been brought to our attention that material in the first three sections of this paper overlaps unpublished material in S. Shelah's dissertation, Hebrew University, Jerusalem (in Hebrew).

\section{REFERENCES}

1. J. Bell and A. Slomson, Models and Ultraproducts, North Holland, (1969).

2. C. C. Chang, A simple proof of the Rabin-Keisler theorem, Bull. Amer. Math. Soc., 71 (1965), 642-643.

3. W. Craig, Relative characterizability and generalized existential quantifiers, Notices Amer. Math. Soc., 9 (1962), 153.

3a. W. Craig and W. Hanf, On relative characterizability in a language, Amer. Math. Soc. Notices, 9 (1962), 152.

4. G. Fuhrken, Skolem-type normal forms for first-order languages with a generalized quantifier, Fundamenta Math., 54 (1964), 291-302.

4a. - Languages with quantifier "there exist at least $\mathbf{\times} \alpha$ ", in The Theory of Models, pp. 121-131, ed. Addison, Henkin, Tarski, North Holland (1965).

5. J. Keisler, The equivalence of certain problems in set theory with problems in the theory of models (abstract), Amer. Math. Soc., Notices, 9 (1962), 339.

6. - Limit ultrapowers, Trans. Amer. Math. Soc., 107 (1963), 382-408.

7. —, Limit ultraproducts, J. of Symbolic Logic, 30 (1965), 212-234.

8. - Logic with the quantifier "there exist uncountably many", Annals of Math. Logic, 1 (1969), 1-100.

9. J. Keisler and J. Silver, End extensions of models of set theory, in Axiomatic Set Theory, (Proceedings of Symposia in Pure Mathematics, Vol. 13 part 1), pp. 177-188, ed. D. Scott, AMS (1971).

10. P. Mahlo, Über linear transfinite Mengen, Berichte über Verh. Königl. Sächs. Ges. der Wiss. zu Leipzig, Math.-Phys. Kl., 63 (1911), 187-225.

11. A. Mal'cev, Small models, Dokl. Akad. Nauk., SSSR 127 (1959), 258-261.

12. M. Morely and R. Vaught, Homogeneous universal models, Math. Scand., 11 (1962), $37-57$.

13. M. O. Rabin, Arithmetical extensions with prescribed cardinality, Indagnationes Math., 21 (1959), 439-446. Ned. Akad. Wet. Proc. Ser. A., 62 (1959) 439-446.

14. P. Vopěnka, A. Pultr, A. Hedrlín, A rigid relation exists on any set, Comment. Math. Univ. Carolinae, 6 (1965), 149-155.

Received October 12, 1970. This work was supported in part by NSF grants GP12209, GP-19405, and GP-19655.

UNIVERSITY OF COLORADO 



\section{PACIFIC JOURNAL OF MATHEMATICS}

\section{EDITORS}

H. SAMELSON

Stanford University

Stanford, California 94305

C. R. HOBBY

University of Washington

Seattle, Washington 98105
J. DugundJI

Department of Mathematics

University of Southern California

Los Angeles, California 90007

RICHARD ARENS

University of California

Los Angeles, California 90024

\section{ASSOCIATE EDITORS}

E. F. BeCKENBACH

B. H. NeumanN

F. WOLF

K. YosHIDA

\section{SUPPORTING INSTITUTIONS}

UNIVERSITY OF BRITISH COLUMBIA

CALIFORNIA INSTITUTE OF TECHNOLOGY

UNIVERSITY OF CALIFORNIA

MONTANA STATE UNIVERSITY

UNIVERSITY OF NEVADA

NEW MEXICO STATE UNIVERSITY

OREGON STATE UNIVERSITY

UNIVERSITY OF OREGON

OSARA UNIVERSITY
UNIVERSITY OF SOUTHERN CALIFORNIA STANFORD UNIVERSITY

UNIVERSITY OF TOKYO

UNIVERSITY OF UTAH

WASHINGTON STATE UNIVERSITY

UNIVERSITY OF WASHINGTON

AMERICAN MATHEMATICAL SOCIETY

NAVAL WEAPONS CENTER

Printed in Japan by International Academic Printing Co., Ltd., Tokyo, Japan 


\section{Pacific Journal of Mathematics}

\section{Vol. 40, No. $1 \quad$ September, 1972}

Alex Bacopoulos and Athanassios G. Kartsatos, On polynomials

approximating the solutions of nonlinear differential equations........

Monte Boisen and Max Dean Larsen, Prüfer and valuation rings with zero

divisors ..........................................

James J. Bowe, Neat homomorphisms

David W. Boyd and Hershy Kisilevsky, The Diophantine equation

$$
u(u+1)(u+2)(u+3)=v(v+1)(v+2) \ldots \ldots \ldots \ldots \ldots \ldots \ldots
$$

George Ulrich Brauer, Summability and Fourier analysis ...............

Robin B. S. Brooks, On removing coincidences of two maps when only one,

rather than both, of them may be deformed by a homotopy ............

Frank Castagna and Geert Caleb Ernst Prins, Every generalized Petersen

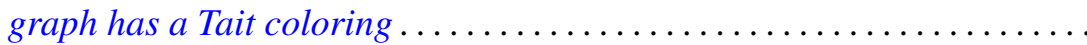

Micheal Neal Dyer, Rational homology and Whitehead products ..........

John Fuelberth and Mark Lawrence Teply, The singular submodule of a

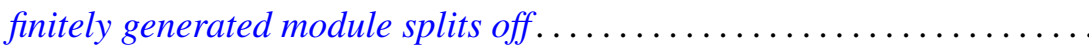

Robert Gold, $\Gamma$-extensions of imaginary quadratic fields ............ 83

Myron Goldberg and John W. Moon, Cycles in k-strong tournaments.......

Darald Joe Hartfiel and J. W. Spellmann, Diagonal similarity of irreducible

matrices to row stochastic matrices...............

Wayland M. Hubbart, Some results on blocks over local fields ..

Alan Loeb Kostinsky, Projective lattices and bounded homomorphisms....

Kenneth O. Leland, Maximum modulus theorems for algebras of operator

valued functions ...

Jerome Irving Malitz and William Nelson Reinhardt, Maximal models in the

language with quantifier "there exist uncountably many" ..

John Douglas Moore, Isometric immersions of space forms in space

forms.

Ronald C. Mullin and Ralph Gordon Stanton, A map-theoretic approach to

Davenport-Schinzel sequences ....................

Chull Park, On Fredholm transformations in Yeh-Wiener space. .

Stanley Poreda, Complex Chebyshev alterations ..............

Ray C. Shiflett, Extreme Markov operators and the orbits of Ryff. ...

Robert L. Snider, Lattices of radicals .....................

Ralph Richard Summerhill, Unknotting cones in the topological

category ................................

Charles Irvin Vinsonhaler, A note on two generalizations of $\mathrm{QF}-3 \ldots \ldots 229$

William Patterson Wardlaw, Defining relations for certain integrally

parameterized Chevalley groups...................

William Jennings Wickless, Abelian groups which admit only nilpotent

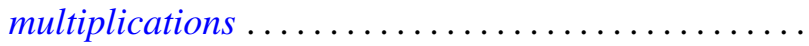

